\title{
Defining Terms of Constitutionally or Randomly Variable Induction of Response to Genotoxic Lesions in Carcinogenesis
}

\author{
Lawrence M Agius* \\ Department Of Pathology, University Of Malta Medical School, Malta
}

Submission: July 22, 2018; Published: August 01, 2018

"Correspondence Address: Lawrence M Agius, Formerly Department of Pathology, Department Of Pathology, Mater Dei Hospital, TALQROQQ, University Of Malta Medical School, Msida, Malta Europe, Tel: 356-21451752; Email: lawrence.agius@um.edu.mt

Abstract

System pathways cooperate with the introduced dimensions of potential repair of genotoxic lesions in an extensive and potential series of adaptive changes or of tumorigenesis within the milieu of further potential progression as induction phenomena in their own right. In such manner the development of breaks or mismatch profiles of the DNA strands cooperate as profile projections as well testified by the evolution of DNA-damage induced apoptotic pathways. The recognition of damaged DNA is further realized as systems specific for the genotoxic lesions and thus are transforming elements within parent families of induction. DNA genotoxic lesions hence create the dimensionally vast array of induced responses to further model the dynamics of reparable or irreparable pathways that attempt re-constitution of DNA lesions in terms of specific repair responses.

\section{Introduction}

The DNA damage-induced apoptosis series of reactivities testify to the emerging concept of self-induced suppression of tumorigenesis that evolves within the contrasting terms of ongoing phosphorylations and de-phosphorylations of such nuclear factors as p53, Abl and caspase-2. DNA single strand breaks are highly related to carcinogenesis and aging [1]. Aldehydes are the main forces inducing DNA damage and inhibit DNA repair in tobacco smoke tumorigenesis [2]. The ongoing series of post-translational molecular changes are confirmed adaptors in terms of DNA-damage sensors and executioners in the evolution of DNA damage-induced apoptosis in a manner that specifically correlates with targeted single-strand versus double-strand DNA breaks or mismatch. Intercellular interactions play an important role in oxidative injury, cell death, and inflammation as induced by ionizing radiation, especially in terms of oxidative signals and intermediate enzymes such as cyclooxygenase-2 [3]. The initiation and transduction of the DNA damage response signaling pathway involves activated ATM (ataxia-telangiectasia mutated) and ATR (ataxia telangiectasia and Rad3-related) and is crucial to DNA damage repair. ATR or ATM inhibition sensitizes tumor cells to chemotherapy [4].

\section{Dimensions of Self-Induction}

Commensurate dimensions of self-induced apoptosis require a period of delay in damage response for the evolutionarily conserved alterations and adaptation of altered gene expression profiles. Cellular senescence is considered a critical physiologic barrier against tumorigenesis [5]. In such manner, further conformational events prove the essentially crucial dynamics borne out by DNA strand breaks in cell adaptation, senescence and survival of the host cells. DNA damage is mediated by radiation, with G2 arrest occurring in virtually all eukaryotic cells, and a mitotic bypass, to ultimately establish potential cellular senescence; ATM, p53 and p21 are crucial in such response [6]. Incremental dynamics further instigate targeting as evidenced and projected by DNA lesions that may persist for long periods of time as temporally-extended pathways of attempted cell adaptation to strand breaks or mismatch lesions. Defective DNA polymerase Eta induces sunlight-sensitivity and skin cancerpropensity disorder xeroderma pigmentosum variant and this potentially depends on upstream E3 ubiquitin ligase Rad18; Chk2 dysfunction is a possible risk factor in sunlight-induced skin carcinogenesis [7].

An ongoing series of adaptive changes includes primarily the induced apoptosis of tumor cells as induced by persistent carcinogenesis or by the genotoxic lesions inflicted by chemotherapeutic agents such as platinum adducts. Ionizing and ultraviolet irradiation are also examples of the induced genotoxic lesion agents in generation of repair mechanisms on the part of the damaged DNA itself, or as further confirmatory 


\section{Cancer Therapy \& Oncology International Journal}

dimensions of response to changes in configuration and adaptation to the lesions in the DNA molecular strands. In spite of the dependence of hepatitis D virus on HBV, HDV and HBV promote liver carcinogenesis by distinct molecular mechanisms; DNA replication, damage and repair point to genetic instability as an important mechanism in HDV hepatocarcinogenesis [8].

\section{Tumorigenesis}

Tumorigenesis thus emerges as response dynamics on the part of the genotoxic lesions of a DNA molecule in ways that are closely allied to adaptation, induced apoptosis in response to DNA damage and also a series of host cell responses such as senescence. Mitochondrial transcription factor A (TFAM) is a key mediator in transcription and maintenance of mitochondrial DNA and may regulate the DNA damage response to mtDNA and the activity of mtDNA encoded cytochrome $C$ oxidase; TFAM may prove a potential target for increased sensitisation of cancer cells to radiotherapy [9]. The inclusive phenomenon of molecular folding and unfolding of nuclear factor molecules is also closely related to nuclear import and export in specific terms of stable intra-nuclear accumulation of such nuclear factors.

Substantially attributed lesions to DNA may prove irreparable as projected by DNA damage sensors within the nuclear compartment. There appears to be collaboration of estrogen receptor beta and p53 tumor suppressor activity in breast cancer cells that may accentuate the importance of ligand-regulated estrogen receptor beta to target p53 activity; this may improve the clinical management of resistant breast disease [10].

As such, further constitutional regions of cooperative dynamics may or may not result in an apoptotic series of responses as well-illustrated by the overtly inducing formulas of genotoxicity. Linker histone H1.2 functions as a physiologic barrier for ATM to target chromatin, and PARylation-mediated active H1.2 turnover is necessary for robust ATM activation and repair of DNA damage [11]. As exemplified by the projected dynamics borne out by such DNA damage, the integrity of the genome is a response-phenomenon related closely to and emanating from systems of realized homeostatic mechanics.

\section{Response Mechanics}

Tumorigenesis is thus a response mechanics that by-passes induced apoptosis in the various dimensional capacities for change adaptation on the part of an established lesion in the cellular DNA complement of the damaged host response. The repair of DNA lesions attests to dynamics of response to a damaged DNA rather than to a homeostatically altered DNA molecule. Bacteria may produce genotoxins and free radicals or affect DNA repair leading to genome damage, cell cycle arrest and apoptosis; a changed or misbalanced bacteriome has genotoxic potential inducing neoplastic disease [12].

In such terms, the dynamics of attempted DNA repair are essentially responses to a variable range of genotoxic lesion and not referable to response as constitutively preserved DNA molecules. In manners that approach overlapping series of DNA response, it is the damaged DNA genome that is capable of repair mechanics in response to ongoing accumulation of genotoxic lesions. Sirtuin 1 (SIRT1), a class III histone deacetylase, is involved in cell proliferation, apoptosis, cell cycling, energy metabolism and DNA repair: SIRT1 is a potential oncogenic factor in breast cancer and may inhibit p53 and activate DNA polymerase delta1 [13].

Such complexity arises in the milieu evolution of a damaged genome such that the further projected dynamics create a highly plastic series of responses as well illustrated by p53 action and response in some of the cells that undergo DNA damage-induced apoptosis. Changes have been reported in signal transductivity and robustness of gene regulatory network in leukaemia subtypes [14]. Incremental dimensions further cooperate as systems of adaptive response as further projected by integral pathways of molecular effectors, as well attested by variability in apoptosis response induced in cells with distinct genotoxic lesions.

\section{Profile Systems}

Systems of profile regulatory function and dysfunction induce further complex integration of variably different and specific genotoxic lesions, as further cooperative action in potential repair mechanisms on the part of damaged DNA. As such, the specific responses on the part of DNA molecules that incorporate lesions are specific adaptive performance of potentially extensive profiles of definite or evolving genotoxic lesions. MicroRNA clusters lead to altered biological functions and are key to the pathogenesis of many disorders including carcinogenesis [15]. Damaged DNA induction of adaptation or alternatively of apoptosis is evidential projection for further cooperative response in the face of active dynamics of genomic dysfunctionality and constitution.

\section{Genomic Instability}

The concept of genomic instability arises in conceptual relevance to systems of damaged response in terms of ongoing variability in response to apoptosis, on the one hand, and as systems of potential tumorigenesis. DeSUMOylation modification is linked to carcinogenesis and tumor progression, including transcription, cell cycling, DNA repair and innate immunity [16].

It is relative to induction processes of highly variable profiles of genotoxic lesions that DNA response is potentially a dimensionally distinct process of highly selective compromise in the face of accumulating intra-nuclear factors that coordinate and affect also cytoplasmically imported molecular profiles. In this regard, the profiles of steady intra-nuclear accumulation of such nuclear factors, such as p53, Abl and caspase-2, potentially induce apoptosis in terms specifically of the irreparable nature of the genotoxic lesions. It is further to such measures of apoptotic cell death that induction pathways recognize effector 


\section{Cancer Therapy \& Oncology International Journal}

mechanisms in their own right as specifically and dynamically projected within the milieu of a highly variable damage to the genome as a whole and to given molecular DNA strands. Smoking may cause methylation and reduce expression of repair genes [17].

\section{Conclusion}

In terms of an evolving tumorigenesis-related series of transforming steps in genotoxicity, the evolutionarily preserved responses of such damaged genome are both constitutionally irreparable or reparable in further confirmatory dimensions of adaptive or apoptotic response. MYCN-p53 interaction leads to regulation of alternative p53 targets that are not regulated in the presence of low MYCN levels; affected are E-boxes of genes crucial to DNA damage responses and cell cycle progression [18]. Proposed range of variability in genotoxic lesions partakes and further contributes to an extensive range of potential adaptive change that may ultimately resolve as tumorigenesis or induced apoptotic cell death. Cell migration through dense tissue or small capillaries may engage and even damage the nucleus, with an effect exerted on cell cycling that potentially impacts carcinogenesis; knockdown of repair factors increases DNA damage independent of cell cycling [19]. The incremental genotoxicity of lesions is characterized in terms of potentially random accumulation of such nuclear factors as evidenced by potential to a highly assorted series of effector pathways in response to DNA lesion heterogeneity. The proposed dimensions of such genomic damage are best defined by the evolving constitutional nature of repair pathways that include the further potential for transforming malignant change.

It is relative to the inherent consequences of such induction mechanisms that adaptive cell change parallels the consequences of irreparable damage in the face of further accumulative DNA lesions as in variable models of attempted constitution. Highly repetitive tandem arrays such as satellite sequences in the centromeric and pericentromeric regions of chromosomes are actively transcribed in cancers, and aberrant expression of satellite RNAs accelerates tumorigenesis through increased DNA damage [20].

The realization of injury to DNA is simple formula construction in the face of an essentially random series of adaptive changes that intrinsically define the transforming events as systems of attempted adaptation in their own right. With regard to such extreme complexity emerges the projected range of effector pathways that include sensor systems for such DNA damage. The inclusion dynamics for cooperative dynamics redefine the response pathways as productive adaptation in the face of an ongoing system pathway of projected, irreparable genomic toxicity. Lesion formulation of the cellular DNA is a highly plastic process that is defining parameter in constitutional response. This allows for the emergence of systems of potential adaptive change leading to a significant increment in tumorigenic dynamics and outcome.

\section{References}

1. Kim HJ, Jang CH (2018) Imaging DNA single-strand breaks generated by reactive oxygen species using a liquid crystal-based sensor. Anal Biochem 556: 1-6.

2. Weng MW, Lee HW, Park SH, Hu Y, Wang HT, et al. (2018) Aldehydes are the predominant forces inducing DNA damage and inhibiting DNA repair in tobacco smoke carcinogenesis. Proc Natl Acad Sci USA 1804869115

3. Farhood B, Boradel NH, Mortezaee K, Khanlarkhani N, Salehi E, et al. (2018) J Cell Commun Signal.

4. Shi Q Shen LY, Dong B, Fu H, Kang XZ, et al. (2018) The identification of the ATR inhibitor VE-822 as a therapeutic strategy for enhancing cisplatin chemrsensitivity in oesophageal squamous cell carcinoma" Cancer Lett 432: 56-68.

5. Zheng H, Huang Q, Huang S, Yang X, Zhu T, et al. (2018) Senescence inducer Shikonin ROS-dependently suppressed lung cancer progression. Front Pharmacol 9: 519.

6. Li M, You L, Xue J, Lu Y (2018) Ionizing radiation-induced cellular senescence in normal, non-transformed cells and the involves DNA damage response: a mini review. Front Pharmacol 9: 522.

7. Tanoue Y, Toyoda T, Sun J, Mustafa MK, Tateishi C, et al. (2018) Differential roles of Rad18 and Chk2 in genome maintenance and skin carcinogenesis following UV exposure. J Invest Dermatol.

8. Diaz G, Engle RE, Tice A, Melis M, Montenegro S, et al. (2018) Molecular signature and mechanisms of hepatitis D virus-associated hepatocellular carcinoma. Mol Cancer Res MCR-18-0012.

9. Zhang R, Wang J (2018) HuR stabilises TFAM mRNA in an ATM/p38 dependent manner in ionising irradiated cancer cells. Cancer Sci.

10. Bado I, Pham E, Soibam B, Nikolos F, Gustafsson JA, et al. (2018) ERbeta alters the chemosensitivity of luminal breast cancer cells by regulating p53 function. Oncotarget 9(32): 22509-22522.

11. Li Z, Li Y, Tang M, Peng B, Lu X, et al. (2018) Destabilization of linker histone $\mathrm{H} 1.2$ is essential for ATM activation and DNA damage repair 28: 756-770.

12. Druzhinin VG, Matskova LV, Fucic A (2018) Induction and modulation of genotoxicity by the bacteriome in mammals. Mutat Res 776: 70-77.

13. Xu Y, Qin Q Chen R, Wei C, Mo Q (2018) SIRT1 promotes proliferation, migration, and invasion of breast cancer cell line MCF-7 by up regulating DNA polymerase delta1. Biochem Biophys Res Commun 502(3): 351-357.

14. Li CW, Lai TY, Chen BS (2018) Changes of signal transductivity and robustness of gene regulatory network in the carcinogenesis of leukaemia subtypes via micro array sample data. Oncotarget $9(34)$ : 23636-23660

15. Kabekkodu SP, Shukla V, Varghese VK, D'Souza J, Chakrabarty S, et al (2018) Clustered miRNAs and their role in biological functions and diseases. Biol Rev Camb Philos Soc.

16. Yang Y, Xia Z, Wang X, Zhao X, Sheng Z, et al. (2018) Small molecular inhibitors targeting protein SUMOylation as novel anticancer compounds. Mol Pharmacol 94(3).

17. Carta CFL, Oliveira Alves MG, de Barros PP, Campos MS, Scholz J, et al. (2018) Screening methylation of DNA repair genes in the oral mucosa of chronic smokers. Arch Oral Biol 92: 83-87. 


\section{Cancer Therapy \& Oncology International Journal}

18. Agarwal S, Milazzo G, Rajapakshe K, Bernardi R, Chen Z, et al (2018) MYCN acts as a direct co-regulator of p53 in MYCN amplified neuroblastoma. Oncotarget 9(29): 20323-20338.
19. Pfeifer CR, Xia Y, Zhu K, Liu D, Irianto J, et al. (2018) Constricted migration increases DNA damage and independently represses cell cycle" Mol Biol Cell 9: mbcE18020079.

Your next submission with Juniper Publishers will reach you the below assets

- Quality Editorial service

- Swift Peer Review

- Reprints availability

- E-prints Service

- Manuscript Podcast for convenient understanding

- Global attainment for your research

- Manuscript accessibility in different formats ( Pdf, E-pub, Full Text, Audio)

- Unceasing customer service

Track the below URL for one-step submission https://juniperpublishers.com/online-submission.php 